\title{
Dengue epidemic management in a developing country
}

Context: Dengue epidemic causes a wide spectrum of illness ranging from mild asymptomatic illness to severe fatal dengue. The cumulative dengue diseases burden has attained an unprecedented proportion in recent times with a sharp increase in the size of the human population at risk. Aims: Use of the WHO criteria (2012) for triage of dengue patients in an epidemic in a developing country with resource crunch of trained manpower and infrastructure. Settings and Design: The study was a retrospective and descriptive study carried out in a tertiary center in New Delhi, India during the dengue epidemic of 2013. Materials and Methods: Triage of dengue cases were done in an outpatient department setting as per latest WHO guidelines 2012 and only those cases with danger signs or severe dengue were admitted. A combination of serological studies involving nonstructural protein 1 antigen, IgM and IgG antibodies were used for diagnosis. Statistical Analysis Used: Statistical analysis was done by SPSS version 19 and $P<0.05$ was considered significant. Results: We studied the clinical, biochemical and serological profile of the inpatients retrospectively. Active participation of parents has to be utilized to augment the monitoring of patients in a developing country with manpower and infrastructure resources crunch. Conclusion: In a developing country like India, with an ever exploding population, an epidemic of dengue causes a severe manpower and resources' crunch. Use of the WHO criteria for triage of dengue patients in an epidemic can help in effective management of dengue cases in an epidemic situation.

Key words: Acute respiratory distress syndrome, developing country, encephalitis, gangrene, resource crunch, severe dengue

Bindu T. Nair, Rama Krishna Sanjeev, Arvind Mishra

Department of Pediatrics, Army College of Medical Sciences, Delhi Cantonment, New Delhi, India

Address for the Correspondence: Dr. Bindu T. Nair, Department of Pediatrics, Army College of Medical Sciences, Delhi Cantonment, New Delhi - 110 010, India. E-mail: binduprakashsanjay@ gmail.com

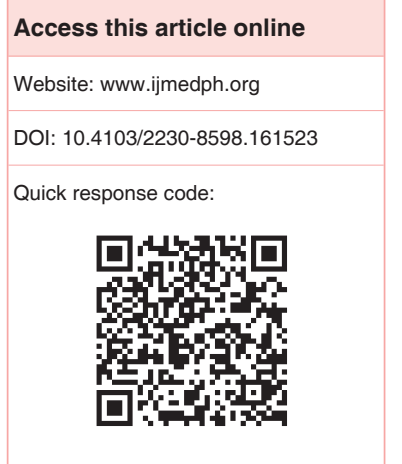

\section{INTRODUCTION}

Dengue fever is a global threat and has become a common epidemic in India especially in the rainy season from July to November. The frequent epidemics of dengue in India are due to multiple factors that include the ever increasing population, increased urbanization, and ineffective vector control especially in the rainy season. ${ }^{[1,2]}$ The lack of availability of a proven vaccine and an anti-viral drug adds to this problem. The diagnosis of dengue fever in endemic areas in developing countries is largely clinical due to costly diagnostic modalities.

WHO has recently issued guidelines in 2012 for management of dengue fever that is more practical than the widely practiced earlier classification of dengue fever and incorporates the wide spectrum of clinical manifestations of dengue. ${ }^{[3,4]}$ The aim of the study was to see the use of the WHO criteria (2012) for triage of dengue patients in an epidemic in a developing country where there is always a resource crunch of trained manpower and infrastructure.

\section{MATERIALS AND METHODS}

The study was a retrospective and descriptive study carried out in a tertiary center in New Delhi, India. This case series was conducted at the Department of Pediatrics in collaboration with the Pathology Department during the peak months of the epidemic of dengue fever in India. Data were collected from the patients admitted with dengue fever from August to November 2013. Triage of dengue cases were done in an outpatient department (OPD) setting as per latest WHO guidelines $2012 .{ }^{[5]}$ The basis of diagnosis was clinical features consistent with dengue-like illness in a setting of 
an ongoing dengue epidemic in New Delhi. WHO clinical guide uses three categories for case management (A, B, C), based on the model of case classification depending on with/without danger signs and severe dengue after a patient has fulfilled the criteria for probable dengue [Table 1].

A combination of serological studies involving nonstructural protein 1 antigen (NS1Ag), IgM and IgG antibodies were used for diagnosis. If the presentation was early (1-5 days), rapid diagnostic kits for NS1Ag alone were used in the cases where clinical suspicion of dengue-like illness was there. In all other cases, NS1Ag, IgM and IgG Ab were done by rapid diagnostic kits to confirm dengue infection. The patients who were suffering from fever and thrombocytopenia due to any chronic illness like aplastic anemia, acute leukemia, hypersplenism and chronic liver disease were excluded from the study.

All patients with warning signs or severe dengue were admitted to pediatrics ward with a physician in attendance. In admitted patients, because of the resources' crunch (number of patients vs. nursing and medical staff) we taught the mothers to report immediately to the staff if the urine output was low. We also used the mother's judgment for assessing child's alertness as an early sign of encephalopathy. A 6 hourly assessment of each inpatient case was done by attending a physician. A detailed clinical monitoring and fluid resuscitation with crystalloids was done in each case in accordance with WHO guidelines. ${ }^{[4]}$ The patients who were triaged and sent back home were also explained about the danger signs and a similar follow-up of urine output by mother was advised. They were followed-up daily on OPD basis.

Clinical data were recorded that included symptoms, signs and laboratory investigations. The judgment to do hematocrit, platelets and electrolytes were done on a case to case basis because if the burden on the hospital laboratory. Patients with severe dengue received intensive care and were catheterized for hourly record of urine output. In children with respiratory distress, arterial blood gases and chest X-ray were used to detect features of acute respiratory distress syndrome (ARDS), respiratory worsening and decision to mechanically ventilate the child. Central venous pressure monitoring was done in critical patients. Monitoring charts and case sheets were reviewed retrospectively and were tabulated for statistical analysis by one of the authors.

Statistical analysis was done by SPSS version 19 (Software package manufactured by IBM Corporation) and $P<0.05$ was considered significant. Ethical approval was obtained from the Institutional Ethics Review Committee.

\section{RESULTS}

The total number of pediatric cases of confirmed dengue fever in our center was 265 over 4 months from August to November 2012 out of which 44 patients were admitted (16.6\%). The youngest patient admitted with confirmed dengue fever was 09 months old who finally succumbed to his illness. This was the only fatality out of the total 265 cases. Seven out of the $44(15.9 \%)$ patients were managed in Intensive Care Unit. The mean number of days at which the inpatients presented with danger signs/symptoms was 5.74 days. 42 out of 44 patients who were admitted had warning signs and 17 out of those had features of severe dengue (38.6\%). Totally, 13 of the patients were females $(29.54 \%)$ and 31 were males $(70.45 \%)$. The numbers of children $<2$ years of age were 4 , between 2 and 5 years were 4 and 36 were $>5$ years of age. The most common symptoms [Table 2] at presentation were fever with rash (59.1\%) followed by associated pain abdomen (45.5\%). Totally, 11 patients presented with frank bleeding $(25 \%)$ in the form of epistaxis, upper gastrointestinal bleeding or hematuria, out of which epistaxis was most common $(8 / 44=18.2 \%)$. Blood transfusion was required in three patients. We transfused single donor platelet in 1 patient only. Of the 44 patients, 17 patients presented with features of shock $(38.6 \%)$ and required fluid resuscitation, predominantly receiving crystalloids. Colloids were used in two of these patients. Seven

\begin{tabular}{|c|c|}
\hline Danger signs & Severe dengue \\
\hline Abdominal pain & $\begin{array}{l}\text { Severe plasma leakage leading } \\
\text { to shock/respiratory distress }\end{array}$ \\
\hline Persistent vomiting & Severe bleeding \\
\hline Clinical fluid accumulation & Severe organ involvement \\
\hline Mucosal bleed & Liver: AST or ALT $\geq 1000$ \\
\hline Lethargy/restlessness & CNS: Impaired consciousness \\
\hline Liver enlargement $>2 \mathrm{~cm}$ & Heart and others \\
\hline $\begin{array}{l}\text { Increase in HCT concurrent with } \\
\text { rapid decrease in platelet count }\end{array}$ & \\
\hline
\end{tabular}

\begin{tabular}{|c|c|c|}
\hline Symptom & Number of cases & Percentage \\
\hline Rash & 26 & 59.1 \\
\hline Pain abdomen & 20 & 45.5 \\
\hline Bleeding manifestation & 11 & 25 \\
\hline Epistaxis & 8 & 18.2 \\
\hline UGI bleed & 2 & 4.5 \\
\hline Hematuria & 1 & 2.3 \\
\hline Neurological & 7 & 15.9 \\
\hline Seizures & 3 & 6.8 \\
\hline Encephalopathy & 4 & 9.1 \\
\hline Cerebral edema & 2 & 4.5 \\
\hline Shock & 17 & 38.6 \\
\hline ARDS & 6 & 13.6 \\
\hline Hepatomegaly & 22 & 50 \\
\hline Splenomegaly & 14 & 31.8 \\
\hline Polyserositis & 23 & 52.3 \\
\hline ASOM & 01 & 2.3 \\
\hline Hemorrhagic bullas & 01 & 2.3 \\
\hline Gangrene & 01 & 2.3 \\
\hline
\end{tabular}

ASOM = Acute suppurative otitis media, ARDS = Acute respiratory distress syndrome, $\mathrm{UGI}=$ Upper gastrointestinal 
patients presented with neurological symptoms (15.9\%), mainly altered sensorium, seizures, features of encephalopathy and cerebral edema. Three patients had generalized tonic clonic seizures $(6.8 \%)$, and two patients had features of cerebral edema (4.5\%). Evidence of serositis was found in 23 cases (52.3\%), and features of shock were found in 17 cases $(38.6 \%)$. Features of plasma leakage coincided with the defervescence period in most of the cases except in 1 case where we experienced features of severe dengue along with persistence of high-grade fever. Six children had features of ARDS and 4 out of them required mechanical ventilation. One child had been weaned off the mechanical ventilatory support and required re-ventilation due to pulmonary edema during the recovery phase. One of the atypical presentations of dengue fever was a child who developed gangrene of distal phalanges of both hands during the course of illness as shown in Figure 1. The onset of gangrene coincided with shock, anuria and fall in platelets. One child with Down's syndrome developed hemorrhagic bullae, severe thrombocytopenia and ARDS not requiring ventilation [Figure 2]. Hepatomegaly was a consistent finding in 22 of these cases (50\%). Splenomegaly was seen in 14 of these patients $(31.8 \%)$. The decision to do a particular test for serology was determined by the timing of presentation as described in material and methods. Both NS1Ag and IgM was done in 10 patients. Patients in whom both NS1Ag and IgM antibody were positive were $9(20.45 \%)$ and in 1 patient NS1Ag was positive but IgM was negative (2.27\%). IgM antibody was positive in 34 patients. One patient was initially managed as severe dengue and had encephalitis and ARDS but was not found to have NS1/IgM positive. However, he exhibited significant rise in $\mathrm{IgG}$ titers on follow-up. Thrombocytopenia and leucopenia were seen in 39 of these patients (88.6\%). Metabolic abnormalities like hypokalemia, hyponatremia and hypoglycemia were seen in 2 patients (4.5\%) each [Tables 2 and 3].

\section{DISCUSSION}

Dengue infection has dramatically increased between 1960 and 2013 by 30 fold. This increase is believed to be due to a combination of

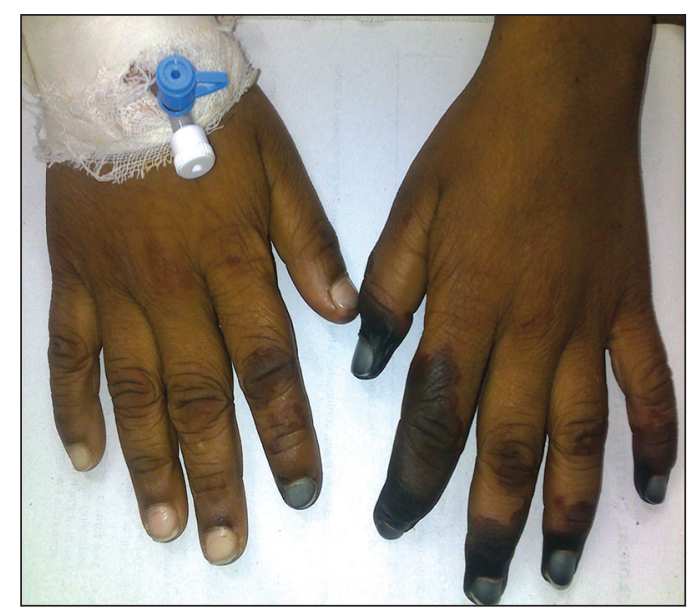

Figure 1: Child who developed gangrene of distal phalanges of both hands urbanization, population growth, increased international travel, and global warming. The geographical distribution is around the equator with $70 \%$ of the total 2.5 billion people living in endemic areas from Asia and the Pacific.

In a developing country like India, with an ever exploding population of 1.26 billion, an epidemic of dengue causes a severe manpower and resource crunch. As the number of dengue cases increase, the bed crunch at public hospitals gets worse. Only those patients who need emergency medical attention are admitted. All public hospitals actively monitor dengue admissions and discharges to manage the bed situation.

Use of the 2012 WHO classification into dengue \pm warning signs and severe dengue has been suggested by WHO for triage of patients during an epidemic. For optimal utilization of the hospital resources, we used the presence of warning signs and severe dengue as the criteria for admission. The presence of NS1 antigen positivity, IgM positivity or IgG seroconversion by rapid tests in a clinical setting consistent with dengue fever was treated as positive. ${ }^{[5,6]}$ The patients were monitored in a high dependency unit and mothers were tasked to monitor 6 hourly urine outputs with knowledge of the normally expected rates.

Parents of children sent back from the OPD too were given instructions on similar lines (particularly for $48 \mathrm{~h}$ postdefervescence). They were asked to report back in the event of any deterioration.

\begin{tabular}{lcc}
\multicolumn{3}{l}{ Table 3: Distribution of lab parameters $(\boldsymbol{n}=\mathbf{4 5})$} \\
\hline Lab parameters & Number of cases & Percentage \\
\hline Thrombocytopenia & 39 & 88.6 \\
Leukopenia & 39 & 88.6 \\
Dengue serology positive & 44 & 100 \\
NS1Ag positive & 22 & 50 \\
IgM Ab positive & 29 & 65.9 \\
IgG Ab positive & 7 & 15.9 \\
Hyponatremia & 2 & 4.5 \\
Hypokalemia & 2 & 4.5 \\
\hline
\end{tabular}

$\mathrm{NS} 1 \mathrm{Ag}=$ Nonstructural protein 1 antigen

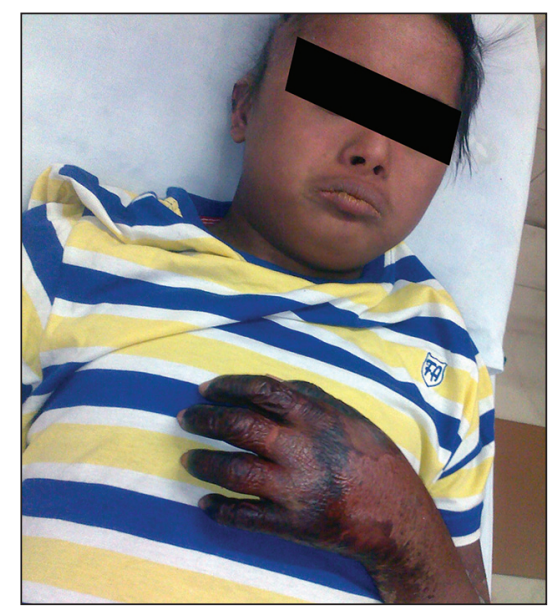

Figure 2: Child with Down's syndrome developed hemorrhagic bullas, severe thrombocytopenia and acute respiratory distress syndrome 
Mothers were the first to report altered behavior and alertness in their children and pick up early evidence of encephalopathy. Children who developed neurological complications recovered fully except in one child in whose case multiple anticonvulsants were used to treat focal seizures due to left frontal gliosis. Similar observations regarding neurological sequelae are noted in earlier reports. ${ }^{[7-10]}$ Only three of the seven with neurological manifestations had shock that is similar to the observations of Kamath and Ranjit. ${ }^{[8]}$ The total mortality among the treated patients was one with a case fatality rate of $<1 \%$.

\section{CONCLUSION}

The management of dengue cases during an epidemic requires a comprehensive team-based approach with round the clock OPD services, well laid out guidelines for triage, laboratory services for diagnosis and blood banking, management of complications, along with availability of intensive care facilities like mechanical ventilation. Active participation of parents has to be utilized to augment the monitoring of patients with shock or incipient shock with urinary output record and to diagnose encephalopathy. Use of the WHO criteria for triage of dengue patients in an epidemic can help in effective management of dengue cases in an epidemic situation.

\section{ACKNOWLEDGMENT}

We would like to acknowledge the Department of Pathology.

\section{REFERENCES}

1. Guzman MG, Halstead SB, Artsob H, Buchy P, Farrar J, Gubler DJ, et al. Dengue: A continuing global threat. Nat Rev Microbiol 2010;8:S7-16.

2. Chakravarti A, Matlani M, Kashyap B, Kumar A. Awareness of changing trends in epidemiology of dengue fever is essential for epidemiological surveillance. Indian J Med Microbiol 2012;30:222-6.

3. WHO. Comprehensive guidelines for prevention and control of dengue and dengue hemorrhagic fever. New Delhi: Regional Office for SouthEast Asia; 2011. p. 1-56.

4. WHO. Handbook for Clinical Management of Dengue. Geneva: WHO; 2012. p. 2-81.

5. Chakravarti A, Kumar A, Malik S. Detection of dengue infection by combining the use of an NS1 antigen based assay with antibody detection. Southeast Asian J Trop Med Public Health 2011;42:297-302.

6. Whitehorn J, Farrar J. Dengue. Br Med Bull 2010;95:161-73.

7. Pancharoen $\mathrm{C}$, Thisyakorn U. Dengue virus infection during infancy. Trans R Soc Trop Med Hyg 2001;95:307-8.

8. Kamath SR, Ranjit S. Clinical features, complications and atypical manifestations of children with severe forms of dengue hemorrhagic fever in South India. Indian J Pediatr 2006;73:889-95.

9. Varatharaj A. Encephalitis in the clinical spectrum of dengue infection. Neurol India 2010;58:585-91.

10. Solomon T, Dung NM, Vaughn DW, Kneen R, Thao LT, Raengsakulrach B, et al. Neurological manifestations of dengue infection. Lancet 2000; 355:1053-9.

How to cite this article: Nair BT, Sanjeev RK, Mishra A. Dengue epidemic management in a developing country. Int J Med Public Health 2015;5:213-6.

Source of Support: Nil, Conflicts of Interest: None declared. 